\title{
TV/Series
}

1 | 2012

Les Séries télévisées américaines contemporaines :

entre la fiction, les faits, et le réel

\section{«Is there a Doctor in the HOUSE ? » : les séries télévisées médicales face au monde réel}

Pamela Tytell

\section{(2) OpenEdition}

\section{Journals}

Édition électronique

URL : http://journals.openedition.org/tvseries/1551

DOI : 10.4000/tvseries. 1551

ISSN : 2266-0909

Éditeur

GRIC - Groupe de recherche Identités et Cultures

Référence électronique

Pamela Tytell, « «Is there a Doctor in the HOUSE ? » : les séries télévisées médicales face au monde réel », TV/Series [En ligne], 1 | 2012, mis en ligne le 15 mai 2012, consulté le 10 décembre 2020. URL: http://journals.openedition.org/tvseries/1551 ; DOI : https://doi.org/10.4000/tvseries.1551

\section{(c) (i) (3)}

$T V / S e r i e s$ est mis à disposition selon les termes de la licence Creative Commons Attribution - Pas d'Utilisation Commerciale - Pas de Modification 4.0 International. 


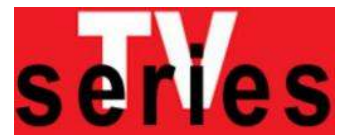

«Is there a Doctor in the HOUSE ? » :

les séries télévisées médicales face au monde réel

Pamela TYTELL

En dépit des exagérations et des raccourcis, les séries télévisées américaines récentes présentent le monde médical à travers une mise en scène réaliste et efficace qui met l'accent sur les détails et la vraisemblance. Des médecins collaborent à chaque scénario, les internautes traquent des erreurs et les acteurs exécutent les gestes techniques avec une précision qui impressionne les spécialistes les plus exigeants. Le succès de la série House, M.D. s'explique par l'identification à l'antihéros, l'utilisation des effets spéciaux et une façon d'aborder la maladie et les traitements qui rappelle "la télévision éducative » (educational television), telle qu'elle est pratiquée aux Etats-Unis.

David Shore n'a rien laissé au hasard. Il a mélangé deux genres : la série médicale et la série policière pour aboutir à un "hospital whodunit » où le Dr. Gregory House agit comme le détective de fiction Sherlock Holmes. D'ailleurs, il y a beaucoup de références aux œuvres d'Arthur Ignatius Conan Doyle dans House, M.D. Après avoir énuméré les raisons du succès de cette série, j'évoque certains comportements des téléspectateurs, notamment leur perception du monde médical. Certains croient que tout se passe comme à la télévision; ils ont une vision fausse du fonctionnement des hôpitaux. J'étudie également les similarités entre la vie des comédiens et celle des personnages qu'ils incarnent dans ces séries médicales.

$\mathrm{L}$ es séries télévisées mettant en scène des médecins et du personnel médical existent depuis les débuts de la télévision. En France, on retrouve plusieurs titres dans la base des données de l'INA dont Janique Aimée (1963), Le Chirurgien de Saint-Chad (1976), Erika Werner (1978), Docteur Teyran (1981), Marine Verdier (1981), Médecins de nuit (1986), etc. Mais, aux Etats-Unis, cette tradition de " medical dramas », ou séries médicales, est plus ancienne. Ce genre télévisuel dramatique, au cours duquel l'action se déroule en milieu hospitalier ou dans un secteur médical, aborde le quotidien professionnel des personnages ainsi que des aspects de leur vie privée. Ainsi, Doctor Kildare et Ben Casey, diffusées de 1961 à 1966, étaient les émissions les plus regardées à l'époque. Majoritairement dramatique, le genre compte aussi des "soap hospital », comme General Hospital, diffusée depuis 1963, ou $M^{*} A^{*} S^{*} H^{*}$ (1972-1983), qui avait ajouté un côté comique au genre. On retrouve aussi Dr. Quinn, Medicine Woman (1993-1998), Chicago Hope (1994-2000) ou E.R. (1994-2009). Parmi les séries médicales diffusées actuellement, il y a Scrubs (depuis 2001), Nip/Tuck (depuis 2003), House, M.D. (depuis novembre 2004), Grey's Anatomy (depuis 2005) et son "spin off " Private Practice (depuis 2007). A cette liste il faut ajouter au moins 
cinquante séries médicales dont les plus connues sont Marcus Welby, M.D. ( 1969-1976), Quincy, M.E. (1976-1983), Trapper John, M.D. (1979-1986), St. Elsewhere (1982-1988), Doogie Howser, M.D. (19891993), Northern Exposure (1990-1995), Providence (1999-2002), Third Watch (1999-2005). On continue de tourner ces séries. En 2009, huit nouvelles séries ont été programmées sur les chaînes américaines : The Listener, Three Rivers, Mental, Royal Pains, Hawthorne, Nurse Jackie, Trauma et Mercy. Po Pourtant, c'est House, M.D., créé en 2004, diffusée dans 66 pays, qui était la série la plus regardée au monde en 2008. Depuis septembre 2010, on peut voir la $7^{\mathrm{e}}$ saison aux Etats-Unis (Fox/NBC). La $6^{\mathrm{e}}$ saison est diffusée en France (TF1) depuis le 19 avril 2011.

C'est un événement personnel qui m'a fait m’interroger sur le succès planétaire de cette série. En mai 2008, à New York, je sortais de l'ascenseur d'une unité de soins palliatifs réservée aux patients en stade terminal d'un cancer. Dans chaque chambre il y avait une grande télévision à écran plasma qui recevait au moins 400 chaînes diffusées sur le câble et par satellite. Dans cette unité toutes les portes restaient ouvertes, sauf lors d'un décès. Pendant que je marchais dans le couloir, je passais devant la porte de douze chambres, avant d'arriver à la chambre occupée par mon père. Je n'entendais que deux choses: les alarmes des machines qui régulaient le débit des produits administrés en intraveineuse et la voix du comédien Hugh Laurie. Tous les téléviseurs, y compris celui dans la salle de repos du staff médical, diffusaient un épisode de House, M.D. Les familles des patients ne regardaient ni une émission de variétés, ni un jeu, ni un documentaire, ni les informations diffusées sur $\mathrm{CNN}$ ou Fox News. Leurs yeux étaient fixés sur le Dr. Gregory House qui travaille dans un hôpital fictif de Princeton, New Jersey, de l'autre côté du fleuve Hudson, à une heure de voiture. Dans cet hôpital, House faisait partie de la vie des familles pour qui il ne restait que la médecine palliative.

Je voulais comprendre cette fascination pour un monde si éloigné d'une unité de soins palliatifs, surtout à travers l'identification au personnage d'un médecin cynique, anticonformiste qui n'éprouve aucune compassion pour ses patients, préférant se concentrer sur leurs symptômes plutôt que de les rencontrer. Certes, depuis Sophocle, le drame et le théâtre permettent de faire face aux pressions de la vie en société, servant de catharsis pour extérioriser les émotions à travers l'observation des personnages et l'identification à leurs destins tragiques. A la fin du spectacle, le spectateur se sent mieux. Certaines séries télévisées se servent de la catharsis. Le fait qu'une série ne reflète pas la réalité, mais tente de s'en rapprocher par le souci du détail ou en nous racontant quelque chose sur notre réalité peut expliquer, en partie, le succès de certaines séries. C'est la raison pour laquelle, en 2011, aux Peoples Choice Awards, la série House, M.D. a gagné la récompense de « Favorite TV Drama». Hugh Laurie a décroché celle de 
«Favorite TV Drama Actor » et Lisa Edelstein celle du « Favorite TV Drama Actress. En fait, très rapidement, j'ai compris que cette série avait franchi un cap, réunissant des ingrédients divers dans une recette efficace qui garantit son succès auprès du public.

Dès 1964, Marshall McLuhan avait prédit le succès de ces « medical dramas »:

One of the most vivid examples of the tactile quality of the TV image occurs in medical experience. In closed-circuit instruction in surgery, medical students from the first reported a strange effect - that they seemed not to be watching an operation, but performing it. They felt that they were holding the scalpel. Thus the TV image, in fostering a passion for depth involvement in every aspect of experience, creates an obsession with bodily welfare. The sudden emergence of the TV medic and the hospital ward as a program to rival the western is perfectly natural ${ }^{1}$.

Aujourd'hui, aux Etats-Unis, ce sont des avocats, des policiers et des médecins qui dominent les séries diffusées en "prime time. » Déjà, en 1982, une étude américaine avait démontré qu'on trouvait cinq fois plus de médecins à la télévision que dans notre vie de tous les jours :

The typical viewer sees about 12 doctors and 6 nurses each week on prime time alone, including 3 doctors and 1 nurse in major roles. By comparison, the same viewer will see only 1 scientist in a week's prime time viewing, and a scientist will be cast in a major role once every 2 weeks ${ }^{2}$.

On peut faire le même constat de ce côté-ci de l'Atlantique. Selon Sabine Chalvon-Demersey, "en France, les professionnels de la santé constituent le deuxième groupe professionnel des héros de fiction, juste après les professionnels de l'ordre public ${ }^{3}$. "

Comment expliquer cette attirance pour des médecins de fiction et des hôpitaux fictifs qui servent de décor à des rebondissements entre chefs de service, spécialistes et internes? Pour le téléspectateur américain, ces médecins symbolisent le pouvoir,

${ }^{1}$ Marshall McLuhan, Understanding Media: The Extension of Man, chap. 31: "Television: The Timid Giant, » Londres, Routledge \& Kegan Paul, 1964 repris par Sphere Books Limited,1967, p. 351.

${ }^{2}$ G. Gerbner, M. Morgan, N. Signorielli, « Programming, health portrayals, what viewers see, say and do, »1982, p. 293 in D. Pearl, L. Bouthilet \& J. Lazar, éds. Television and Behavior, vol. 2 Technical Reviews, Rockville, Maryland, p. 291-307.

3 Sabine Chalvon-Demersey, «La Confusion des Conditions : une enquête sur la série télévisée Urgences, » Réseaux, 95, 1999, p. 241 (p.235-283). 
l'autorité et le savoir. Pourtant, le téléspectateur n'est pas dupe. Il sait parfaitement qu'il y a des inexactitudes, des exagérations ou des raccourcis. Dans ces séries on ne respecte pas le secret médical. On ne recherche pas le consentement des patients avant de procéder à des actes médicaux ou d'avoir recours à des techniques de soins expérimentales. On présente un monde médical éloigné de la réalité. Cependant, ce qui intrigue c'est la réalité telle qu'elle est perçue, «the perceived reality » et la façon dont cette exposition au monde médical modifie le comportement du téléspectateur. Il sait qu'il regarde une fiction, mais la présentation et la mise en scène réalistes ont modifié sa perception et son implication ( « involvement ») dans l'intrigue, surtout quand il regarde une série qui soulève des questionnements philosophiques d'éthique ou de déontologie médicale en abordant des thèmes polémiques tels l'avortement, l'euthanasie, l'acharnement thérapeutique ou les informations à fournir au patient et à sa famille avant la mise en place des traitements. Pour le téléspectateur, ces fictions médicales racontent quelque chose d'essentiel sur sa propre situation personnelle, qu'il reconnaît à l'écran.

\section{Scénario et vraisemblance}

Il faut se rendre à l'évidence: les impératifs du format télévisuel sont incompatibles avec les bases scientifiques de la médecine. Dans une série télévisée il y a une rapidité d'exécution, un nombre excessif de cas rares et une exagération peu conformes à la réalité. Tout se passe dans une effervescence qui serait impossible à gérer au quotidien dans un hôpital. Cependant, les scénaristes nous font oublier ces incompatibilités. Certaines séries, telles E.R ou House, M.D., s'appuient sur un dispositif d'unité de temps, de lieu et d'action qui évoque les pièces de théâtre. Les patients défilent et les médecins les soignent de façon réaliste. Les images sont convaincantes et la partie médicale est crédible. De véritables médecins collaborent à l'écriture des épisodes, renforçant cette impression de proximité et de vraisemblance. Les scénaristes ne prétendent pas refléter la réalité du milieu hospitalier. Ils tentent simplement de s'en rapprocher le plus possible en mettant l'accent sur les détails. Par exemple, l'association des médecins d'urgence, l'ACEP, apportaient précisions et conseils à la série E.R., créé par Michael Crichton, titulaire lui-même d'un diplôme de médecine, même s'il n'a jamais exercé. Crichton avait insisté pour que le matériel médical (stéthoscopes, thermomètres, appareils de radiologie) soit authentique. Les armoires à pharmacie étaient remplies de vraies boîtes de médicaments. Des infirmières, présentes sur le plateau de tournage, montraient aux comédiens les gestes qu'ils devaient reproduire. La série E.R. a également utilisé la référence au documentaire et au reportage d'actualité pour donner l'illusion d'une 
certaine réalité. Cette logique a été poussée très loin car le premier épisode de la dernière saison a été tourné sous forme de documentaire dans les conditions du direct. La scénariste Tracy Stern organisait même des «soirées des infirmières" à la Warner Bros. car les infirmières sont « précieuses pour analyser les paramètres humains qui entrent en jeu dans les situations de crise ${ }^{4}$. " Aujourd'hui, une équipe de médecins collabore à l'écriture de Grey's Anatomy pour que les gestes et la terminologie soient précises. C'est l'attention aux détails qui fait la différence 5 .

Quant au Dr. Ronald Green, professeur de psychiatrie à la faculté de médecine de Dartmouth College, il conseillait les scénaristes de la série The Sopranos et indiquait les médicaments que la psychanalyste Dr. Melfi devait prescrire à son patient sociopathe Tony Soprano. Il avait avoué au New York Times qu'en dépit de son souci d'exactitude concernant les ordonnances, il lui arrivait de se tourner vers d'autres séries télévisées pour des conseils en pharmacologie. Par exemple, quand un personnage a tenté de se suicider en avalant des somnifères, avec la moitié d'une bouteille de Vodka, le psychiatre ne se souvenait plus de ce qu'il fallait faire pour sauver la patiente. Il a suggéré l'administration des perfusions de dopamine parce qu'il avait vu Mark Greene ou Kerry Weaver faire cela dans E.R. Ainsi, le professeur en psychiatrie se tournait vers la série $E . R$. pour obtenir des précisions sur le traitement approprié, car il ne s'agissait pas de sa spécialité médicale 6 .

En ce qui concerne House, M.D., c'est Lisa Sanders, Clinical Professor à Yale University School of Medicine qui est conseillère technique et c'est sa chronique "Diagnosis », publiée dans The New York Times, qui a inspiré la série. Trois médecins vérifient chaque scénario et une infirmière, Bobbin Bergstrom, est conseillère médicale pendant le tournage ou bien joue elle-même le rôle d'une infirmière.

Différentes catégories de téléspectateurs interviennent également dans ces séries. Hormis les sites internet qui traquent des erreurs - toujours l'attention au moindre détail - nous savons que de véritables infirmières et médecins jouent à indiquer le traitement approprié avant que les personnages ne le disent pendant l'épisode. Ainsi, ils écrivent aux scénaristes quand ils remarquent un décalage trop important entre la réalité et la fiction. Par exemple, au début de la série E.R., après que des massages cardiaques ranimaient

\footnotetext{
4 Sarah Sepulchre, "Urgences, décembre 2002 ", Aux Frontières des Séries <www.afds.tv/spip.php?page=imprimer\&id_article=61>, consulté le 12 janvier 2012.

5 Le titre de la série repose sur un jeu de mots : une référence au personnage Meredith Grey, interne en chirurgie, et au texte médical Henry Gray's Anatomy of the Human Body, appellé Gray's Anatomy, publié en 1858, considéré comme "la bible » de l'anatomie humaine par les médecins anglophones.

6 Sarah Boxer, «Therapists go crazy for Tony Soprano, » The New York Times, 29 décembre, 2001.
} 
miraculeusement des patients en arrêt cardiaque, le New England Journal of Medicine a réclamé aux scénaristes davantage de réalisme, car le taux de guérisons est assez faible après une défibrillation. Les scénaristes d'E.R en ont tenu compte. Suite à cet incident, des médecins ont envoyé aux scénaristes des anecdotes et des conseils qui ont rendu la série crédible et mieux acceptée par le corps médical. Ici, la réalité médicale a influencé l'écriture du scenario7.

Qu'en pensent les médecins en France? Il paraît que la véracité des scènes arrive à en rebuter un certain nombre. Interrogée sur les séries médicales, une interne a confié que «les questions que l'on pose aux internes [...] sont exactement celles que l'on peut poser à des internes en France, donc je révisais avec Urgences (E.R.) Mais j'avais tellement l'impression de me retrouver au travail que j'ai arrêté. "Selon un autre interne: "tout est parfaitement crédible, jusqu'au moment où des internes ouvrent des thorax au beau milieu des urgences ; ça, ce n'est pas plausible ${ }^{8}$.»

Le Dr. Yohann Derhy, chirurgien et ancien chef de clinique à l'hôpital Saint-Louis à Paris, regardait Chicago Hope quand il préparait son concours d'internat. " J'étais bluffé à chaque épisode : c'est très précis, ils n'en font pas trop. Grey's Anatomy se défend bien également en matière de crédibilité médicale [...] Tout reste vrai et professionnel, mais c'est plus abordable (pour ceux qui ne sont pas médecins). Quant à Nip/Tuck c'est vraiment n'importe quoi, » s'énerve le chirurgien plasticien. "Sur le plan technique tout est [...] foireux. Ils font des fautes impardonnables, notamment en matière d'asepsie [...]. Même leur gestuelle n'est pas au point. Les images sont particulièrement gore [...] Je peux vous assurer que ce n'est pas comme ça! C'est une caricature poussée à l'extrême. En revanche, pour les termes médicaux et les interventions préconisées pour chaque cas, il n'y a rien à dire [...]. Le cancre, c'est Scrubs, mais c'est normal, l'aspect médical est vraiment anecdotique, » souligne le Dr. Derhy9. Cet avis n'est pas partagé par tout le monde, car Adeline, elle, aime beaucoup Scrubs : « c'est super rigolo, mais absolument pas crédible médicalement parlant ${ }^{10}$. » En ce qui concerne E.R., Dr. Derhy trouve que « les acteurs ont de vrais gestes de médecins professionnels. Cela a dû leur prendre beaucoup de temps pour les reproduire avec tant de précision ${ }^{11}$. " Dans l'ensemble, les médecins français sont sensibles aux efforts de crédibilité de la part des scénaristes américains. Dr. Yohann Derhy pense même que ces

\footnotetext{
7 Voir Dr. Benoit Papon, adaptateur français de la série E.R., cité par Jean-Michel Bader, «Quand Dr. House influence les malades, » Le Figaro.fr, 9 septembre 2009.

8 «Les séries TV Médicales: des termes et des gestes très précis, 》 <www.linternaute.com/santé/magazine/dossier/series-tv-medicales>, consulté le 12 janvier 2012

9 Ibid.

${ }^{10}$ Ibid.

${ }^{11}$ Ibid.
} 
séries ont développé un plus grand respect pour la profession médicale, permettant de rapprocher les médecins du public ${ }^{12}$.

Sabine Chalvon-Demersey est arrivée à la même conclusion. Dans le cadre de son enquête sur la série E.R., elle avait interrogé des membres du personnel médical français afin de préciser « les éléments qui avaient pu créer cette impression de réalité et les raisons qui les avaient attachés à la série ${ }^{13}$. » Les réponses mettaient l'accent sur :

\begin{abstract}
[...] l'exagération, pour tout ce qui concerne l'arrivée des patients et sur l'exactitude pour tout ce qui concerne leur prise en charge [car] le nombre de patients est [...] excessif. L'accent mis sur les situations dramatiques [...] conduit à oublier la dimension [...] plus triviale de l'urgence ordinaire. [...] Le nombre de cas où le pronostic vital est en jeu est très supérieur à celui d'une situation normale [...] et la répartition des pathologies est plutôt fantaisiste [...]. Mais en même temps, tous sont sensibles à l'exactitude et à la précision technique de tout ce qui renvoie à la partie proprement médicale [...]. Le personnel médical apprécie surtout la qualité de la description, presque ethnographique, de la division du travail, des rapports de hiérarchie,[...] de la logique de fonctionnement interne du monde hospitalier (et) la précision technique de la terminologie [...]. Les conditions de travail sont aussi précisément décrites [...]. Notamment tout ce qui tient à la fatigue physique et nerveuse, liée aux changements de rythme et au manque de sommeil [...] et la densité des échanges ${ }^{14}$.
\end{abstract}

Ainsi, la validité scientifique, la justesse des interactions, le souci d'exactitude, qui caractérise aussi la description du rapport aux malades, la présentation réaliste, l'imprévisibilité des dénouements, la faillibilité des personnages, la dimension morale, les échecs de la vie privée, etc. expliquent en partie l'attirance, l'identification et la compassion du téléspectateur.

\title{
2. Lieux de tournage et images
}

La perception de ce monde hospitalier passe aussi par le choix des lieux de tournage et des images. Parfois on tourne dans un hôpital fictif, mais la situation a évolué depuis les années 1990. La série E.R. avait fait le choix de la crédibilité et de l'exactitude en tournant des scènes dans un hôpital désaffecté de Linda Vista près de San Diego. Par la suite, le décor a été entièrement reconstruit dans les studios de la Warner Bros. à Burbank, près de Los Angeles. Cependant, comme le nom de l'hôpital où se situe l'action est réel, l'ancien Cook County, à

\footnotetext{
12 «Les séries TV Médicales: Les patients ont changé, » www.linternaute.com/santé/magazine/dossier/series-tv-medicales consulté le 12 janvier 2012.

${ }^{13}$ Sabine Chalvon-Demersey, op.cit., p. 256

${ }^{14}$ Ibid., p. 256-259.
} 
Chicago, de nombreuses scènes ont été tournées dans cette ville, surtout en hiver, quand Chicago se trouve sous la neige ${ }^{15}$. Grey's Anatomy se sert du VA Sepulveda Ambulatory Care Unit à North Hills en Californie pour les scènes à l'hôpital fictif, Seattle Grace. Le tournage de la plupart des épisodes de House, M.D. a lieu près de Los Angeles, à Century City, mais des épisodes ont également été tournés en dehors des studios, sur un campus universitaire : the University of Southern California. Les scènes où House est interné dans le Mayfield Psychiatric Hospital, pendant la sixième saison, ont été tournées dans les bâtiments abandonnés de Greystone Park Psychiatric Hospital, à Parsippany-Troy Hills dans le New Jersey ${ }^{16}$, et les prises de vue extérieures de l'hôpital fictif Princeton-Plainsboro sont tournées sur le campus de Princeton University.

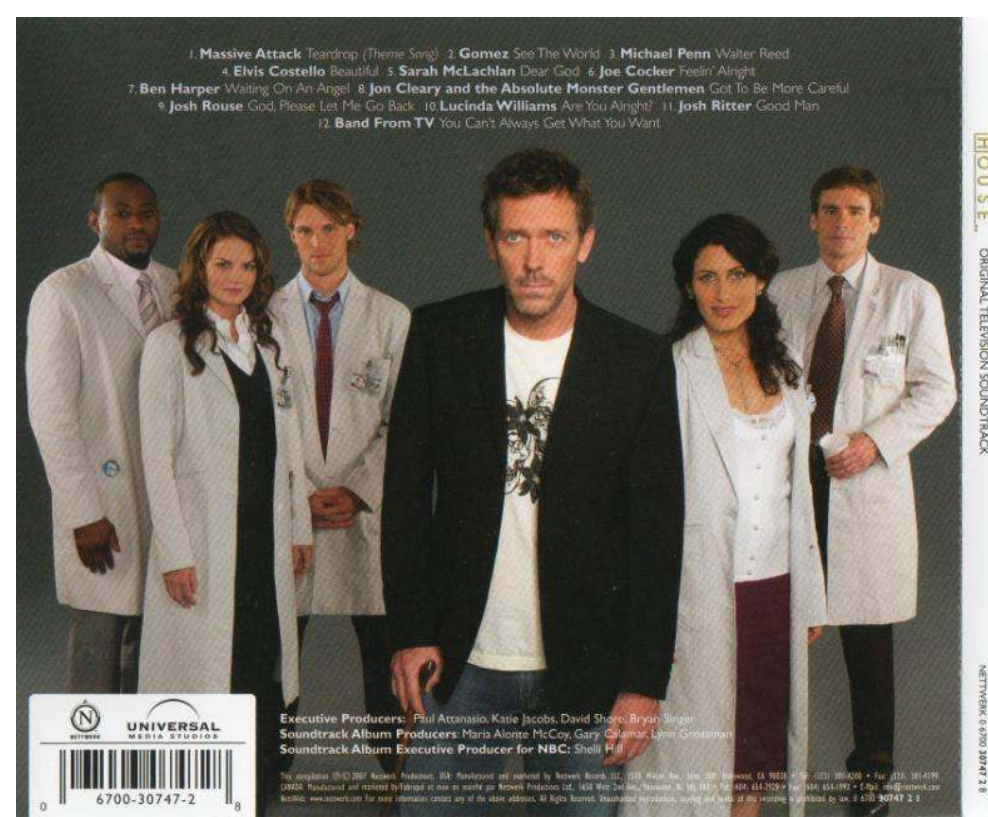

Figure 1 Les membres de l'équipe sont réunis sur une photo qui figure sur l'«Original Television Soundtrack» de la série House, M.D. On y trouve le "theme song»: "Teardrop», du groupe Massive Attack, des morceaux de Gomez, Michael Penn, Elvis Costello, Sarah McLachlan, Joe Cocker, Ben

15 « Les séries TV médicales: images et décors très américains, » $<$ www.linternaute.com/sante/magazine/dossier/series-tv-medicales>, consulté le 12 janvier 2012

${ }^{16}$ Katherine Santiago, « TV show 'House' to film at Greystone Park Psychiatric Hospital, » in New Jersey.com, 14 April 2009. <www.nj.com/news/index.ssf/2009/04>, consulté le 12 janvier 2012. 
Harper, Jan Cleary and the Absolute Monster Gentlemen, Josh Rouse, Lucinda Williams, Josh Ritter, ainsi que "You can't always get what you want", interprété par Hugh Laurie et son groupe Band from TV ${ }^{17}$.

Cette présentation réaliste et ce souci d'exactitude commencent dès le générique, utilisé pendant les saisons 1 à 6 , que l'on peut visionner sur YouTube ${ }^{18}$. Dans la séquence, on retrouve les trois versions différentes des « opening sequences » utilisées en Europe, aux Etats-Unis et à Singapour, en raison des droits d'auteur exigés par le groupe Massive Attack pour leur titre «Teardrop. " En général, l'ancienne séquence d'ouverture commençait par une image tirée d'un IRM cérébral avec le logo $\mathrm{H}$ - symbole international de l'hôpital suivie d'une vue aérienne de l'hôpital fictif. Il y avait également des planches d'anatomie humaine, des modélisations graphiques des ramifications des neurones, l'image d'une cage thoracique aux rayons $\mathrm{X}$ et un aperçu de l'équipe qui marchait dans le couloir derrière Gregory House - une référence au générique de Law \& Order - pour nous mettre dans l'ambiance des séances de diagnostic différentiel qui se répétaient jusqu'à ce que la situation du patient devienne critique ${ }^{19}$.

Les effets spéciaux et les images de synthèse en $3 \mathrm{D}$ du générique nous renvoient aux progrès réels de l'imagerie médicale et son voyage fantastique dans les poumons, les veines, les muscles, voire le cerveau des patients. Ces mêmes images sont toujours présentes dans le nouveau générique de la septième saison mais sont complétées par d'autres images réalistes qui explorent davantage le corps blessé et malade tel qu'on le voit dans Dexter, Bones ou $C^{2} I^{20}$. Certes, la tête et le cerveau ne changent pas : c'est toujours David Shore. Ce nouveau générique a donné lieu à de nombreuses parodies ${ }^{21}$.

\section{Dr. Gregory House, M.D. face au téléspectateur}

\footnotetext{
${ }^{17}$ House, M.D., Original Television Soundtrack, Universal Media Studios, 2007. "All 3 House MD openers/themes/intros de TheRLWS » $<$ http://youtube.com/watch?v=x5i5ERDE_2E\&feature=youtube_gdata_player $>$, consulté le 12 janvier 2012

${ }^{19} \mathrm{La}$ scène d'aviron sur le lac est un clin d'œil au père de Hugh Laurie, médaillé d'or aux Jeux Olympiques (1948) en aviron. C'était également le sport préféré de Hugh Laurie pendant ses études à Cambridge University On peut trouver des informations sur Hugh Laurie et la série dans Paul Challen, The House that Hugh Laurie built: An Unauthorized Biography and Episode Guide, Toronto, ECW Press, 2007; Ian Jackman, House,M.D.: The Official Guide to the Hit Medical Drama, New York, it Books/HarperCollins, 2010. ${ }^{20}$ « House MD Season 7- New Opening Sequence de housefansite», <http://www.youtube.com/watch?v=cGvENehHKow\&feature=youtube_gdata_player > consulté en janvier 2012

${ }^{21}$ On peut visionner ces parodies sur YouTube, notamment celles mettant en scène Sponge Bob et Homer des Simpsons. "SpongeBob MD » <http://www.youtube.com/watch?v=TftTondzbFY>; $>$ et HomerMD » $<$ http://www.youtube.com/watch?v=Zxho8ZDlECw>, consultés en janvier 2012.
} 
Tous ces éléments contribuent au succès phénoménal de la série médicale House, M.D. Mais depuis mon expérience dans l'unité des soins palliatifs d'un hôpital new yorkais, je souhaitais mieux comprendre les ingrédients de cette recette efficace qui garantit le succès planétaire d'une série médicale. Le premier ingrédient est, sans aucun doute, la présence de l'antihéros. La télévision a tendance à construire et à perpétuer des stéréotypes. Le personnage de Gregory House casse le stéréotype étroit et figé du médecin idéal, le héros fort et courageux. On est loin du cliché du chirurgien bronzé dans Nip/Tuck roulant dans une voiture de sport hors de prix. House est l'antihéros boiteux, plein de doutes et de défauts, qui se sert de son expérience et de l'observation des symptômes pour construire des hypothèses, qu'il expérimente avec examens et traitements, pour les réfuter ou les valider, avant d'aboutir à un diagnostic final. Il utilise des méthodes peu conventionnelles et il a souvent des problèmes avec la loi ou la déontologie médicale, car il n'oublie jamais qu'il a lui-même été victime d'un diagnostic erroné. C'est la raison pour laquelle il marche avec une canne, soulage sa douleur en prenant du Vicodin, un analgésique opiacé à base de paracétamol et d'hydrocodone, auquel il est devenu accro. Et il consomme beaucoup d'alcool. Dans son « Poster Paper», préparé pour le colloque sur «Les séries télévisées américaines contemporaines : entre la fiction, les faits et le réel, » à l'Université Paris-Diderot, en mai 2011, Alexis Pichard va encore plus loin quand il écrit que House est un " antihéros machiavélique », car la fin justifie les moyens.

Dans Nurse Jackie (Showtime, juin 2009-), le personnage joué par Edie Falco est également dépendante au Vicodin. Cette infirmière, qui travaille aux urgences de All Saints' Hospital à New York, est obstinée et brillante, mais elle a besoin de drogues diverses pour l'aider à supporter sa vie professionnelle agitée et sa vie personnelle désastreuse. The New York State Nurses Association s'est plainte du comportement du personnage de Jackie Peyton qui viole le code de déontologie de façon répétée, ne respectant pas l'éthique médicale ${ }^{22}$. Pourtant, selon le comédien Hugh Laurie, il ne semble pas y avoir de plainte concernant les viols répétés de la déontologie médicale par Gregory House, hormis les problèmes du personnage principal avec la justice dans la série elle-même. Une douleur physique serait-elle plus légitime qu'une douleur psychologique?

${ }^{22}$ Voir le site de la série Nurse Jackie

<www.sho.com/site/nursejackie/home.do>, consulté en janvier 2012. 


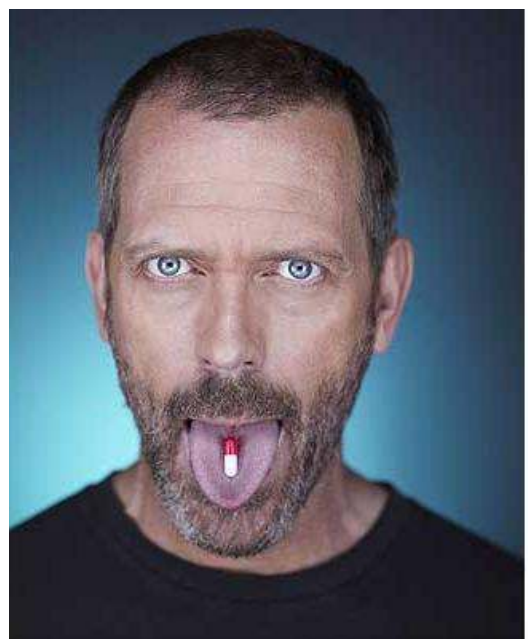

Figure 2 La dépendance de G. House à la Vicodin joue un rôle important dans le scenario. Il utilise d'autres analgésiques puissants tels que la morphine, l'oxycodone ou la méthadone.

Hormis le fait que House, M.D., ne soit pas comme les autres séries, où on court dans les couloirs de l'hôpital derrière des " gurneys », ces brancards sur roues qui transportent des patients, la présence d'un deuxième ingrédient permet d'expliquer le succès de la série: la pédagogie télévisuelle. House propose une nouvelle façon d'aborder la médecine. On entre dans le domaine de la télévision éducative, educational television au sens américain du terme, c'est-àdire une émission qui reproduit et transmet des représentations du savoir dans un format de fiction à travers un divertissement. Cette série informe, distrait et enseigne dans le cadre d'un format répétitif qui permet au téléspectateur de se concentrer sur le contenu. Le téléspectateur croit participer à la résolution de l'énigme médicale. Les maladies existent vraiment, même s'il s'agit de cas rares. Pour trouver le bon traitement, House passe en revue plusieurs hypothèses, même si on a l'impression que son intuition relève davantage de l'art divinatoire que de la médecine. Les effets spéciaux et les images de synthèse en $3 \mathrm{D}$ font le reste du travail en nous amenant dans des voyages fantastiques à travers ce corps humain qu'on aperçoit dès le nouveau générique. Ces effets spéciaux et images en $3 \mathrm{D}$ représentent, reconstruisent et expliquent le monde médical de façon simple. Tout en restant passif, le téléspectateur - qui est de plus en plus sophistiqué grâce à l'accès facilité aux nouvelles technologies - apprend, interprète et assimile des informations médicales, se familiarisant avec la terminologie, les symptômes, le diagnostic, les analyses, les traitements, l'éthique et 
même les demandes intellectuelles de ce métier. Sur le site internet de la série, on détaille par le menu tous les cas rarissimes abordés au fil des épisodes. De quoi en connaître un rayon en maladies rares ! Et on demande même aux internautes de signaler des erreurs ${ }^{23}$. Andrew Holtz, ancien journaliste médical à CNN, a même consacré un livre à l'explication médicale des énigmes résolues dans la série ${ }^{24}$. Les Américains apprennent le droit à travers les séries télévisées, en écoutant les avocats et les policiers dans des situations proches de la réalité. Ils apprennent la médecine en observant les médecins à l'œuvre dans les séries médicales telles E.R. et House, M.D.

Le troisième ingrédient est le mélange de deux genres, la série médicale et la série policière, permet aussi d'expliquer pourquoi la démarche de l'orgueilleux et brillant médecin (diagnosticien hors pair, mais aussi caustique et antisocial) relève autant du policier et de la psychanalyse que de la médecine. En fait, House, M.D., est une série policière se déroulant dans un milieu médical. Nous sommes en présence d'un «whodunit », une forme complexe du roman policier dans laquelle la structure de l'énigme est le facteur prédominant et le lecteur dispose des mêmes indices que l'enquêteur. House, M.D. est un " hospital whodunit », où les médecins recherchent les symptômes et leurs causes dans une enquête criminelle digne de Scotland Yard. Le service de diagnostic de Princeton-Plainsboro est chargé de résoudre un mystère médical posé par la santé d'un seul patient, à la différence d'E.R. et de Grey's Anatomy où l'intrigue porte sur plusieurs patients pendant chaque épisode. D'ailleurs, les images de synthèse dans House, M.D. donnent souvent l'impression de regarder une série policière comme C.S.I. ou N.C.I.S. : House et son équipe recherchent la vérité et le traitement approprié en partant sur les traces des indices menant à la découverte de l'origine de la pathologie, tout comme Grissom et son équipe dans C.S.I., ou Gibbs et son équipe dans N.C.I.S., qui recherchent des indices pour identifier le meurtrier en fouillant dans l'appartement du suspect, victime ou patient. La construction de ces séries - à base d'enquêtes - se déroule en quatre phases : hypothèse, échec, nouvelle hypothèse, résolution de l'énigme. Avec Gregory House, la traque du virus (et du lupus !) remplace celle du criminel. L'enquête est retardée, ensuite réorientée par le mensonge et la dissimulation de symptômes/indices dans l'histoire personnelle du patient ou dans celle de ses proches, d'où la réplique culte ou Houseism : «Everybody lies » («Tout le monde ment »). La science médicale dans House et les méthodes médico-légales dans C.S.I., obligent les scénaristes à mélanger cas criminels, cas médicaux

${ }^{23}$ Parmi les nombreux sites consacrés à la série House, M.D., on peut consulter le site officiel <http://www.housemd-guide.com>, consulté en janvier 2012.

${ }^{24}$ Andrew Holtz, The Facts Behind the Addictive Medical Drama: The Medical Science of House, M.D., New York, Berkeley Boulevard Books et New York, Penguin Books, 2006. 
et fiction. Parfois, le téléspectateur ne parvient plus à séparer le vraisemblable du faux, parce que les scénaristes se concentrent sur les méthodes scientifiques de pointe dans C.S.I. et les traitements médicaux peu conventionnels dans House, M.D.

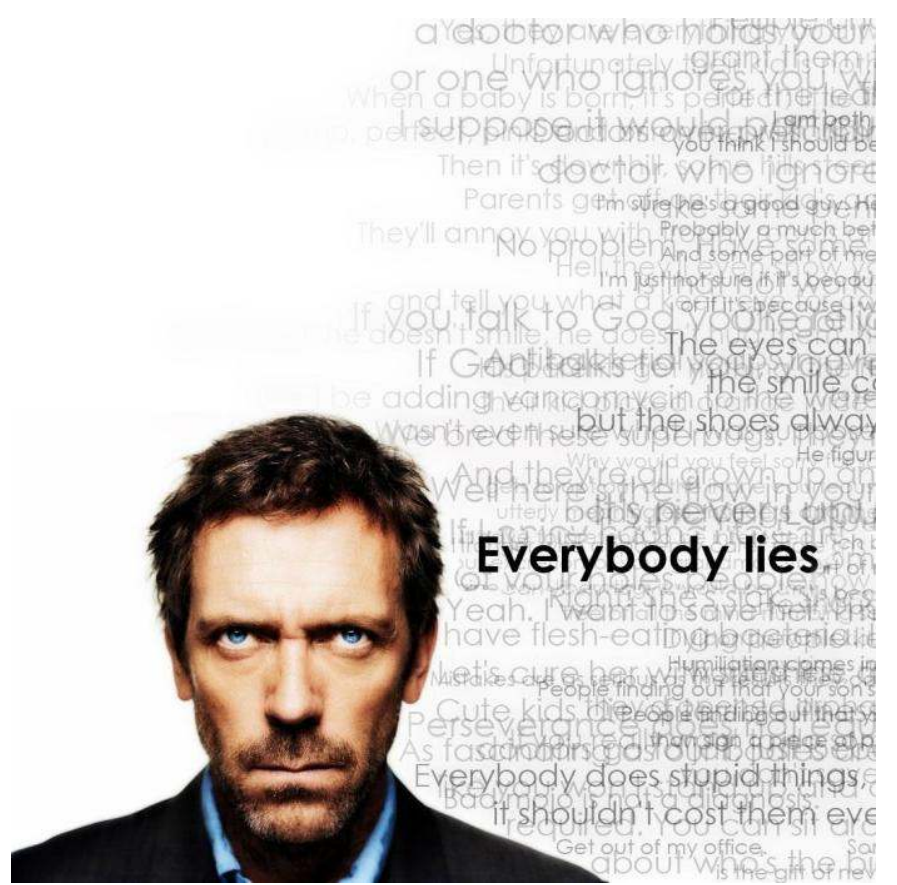

Figure 3 Montage des répliques cultes («Houseisms ») qui figurent sur les accessoires en vente dans le magasin des Studios NBC

\section{House, M.D. et la culture de l'enquête}

Quand on évoque l'enquête criminelle de fiction on pense tout de suite au roman policier et au premier détective de fiction, le chevalier C. Auguste Dupin, le personnage créé par l'écrivain américain Edgar Allan Poe (1809-1849) pour sa trilogie. Malgré la présence de Dupin dans seulement trois nouvelles de Poe : The Murders in the Rue Morgue (1841), The Mystery of Marie Roget (1842-1843) et The Purloined Letter (1844), ces nouvelles - traduites en français par Baudelaire - sont à la base du roman policier moderne et ont inspiré l'ensemble des enquêtes au cours desquelles les seules facultés d'observation et d'analyse permettent de résoudre des énigmes criminelles. Les Ecrits de Jacques Lacan s'ouvrent sur un texte de 
cinquante pages, une étude de The Purloined Letter ${ }^{25}$. Ce n'est pas un hasard si Jacques Lacan a choisi La Lettre volée pour expliquer le sens de ses théories psychanalytiques. Il existe plusieurs points communs entre la psychanalyse et ce texte de Poe, précurseur du roman policier : d'abord, la présence d'une énigme et une accumulation d'indices ; puis le recours aux pulsions inconscientes (pulsion de mort, culpabilité refoulée, etc.) pour structurer l'intrigue criminelle ; ensuite le travail de détective, qui réunit les indices et saisit la logique propre à ce qui est non-formulé, donnant ainsi un langage à ce qui n'est qu'une accumulation d'images vagues; enfin, l'explication de plus en plus élaborée, l'irruption soudaine de la vérité, et la résolution de la crise. On est très proche du travail du psychanalyste qui, dans sa quête pour élucider un mystère, se penche sur le discours de son analysant, évalue chaque élément de façon minutieuse et systématique et tire des conclusions à partir de l'observation et de l'étude des indices ${ }^{26}$.

Dans son autobiographie, Sir Arthur Ignatius Conan Doyle (1859 - 1930), écrivait que le détective talentueux de Poe faisait partie de ses héros depuis l'enfance et l'avait inspiré pour la création du personnage de Sherlock Holmes ${ }^{27}$. Nous savons que Doyle connaissait les procédés de l'enquête policière, les travaux d'Alphonse Bertillon à l'identité policière de Paris (1893), et l'administration de la preuve des crimes par le moyen de recoupements rationnels, de constatations et de témoignages. Mais Doyle s'est inspiré de deux modèles pour créer son célèbre détective : le chevalier C. Auguste Dupin, le personnage de Poe, et Joseph Bell, son ancien professeur, célèbre chirurgien écossais, dont le pouvoir d'observation et la finesse de diagnostic furent légendaires au XIXe siècle. Le jeune médecin Doyle travaillait comme l'assistant de Bell. Dans une lettre de 1892, Arthur Conan Doyle informa Joseph Bell que «le travail analytique de Holmes n'était pas plus impressionnant que les effets produits sur les patients de Bell ». Partant du noyau constitué d'éducation, d'observation et de conclusions inculqués à ses élèves, Arthur Conan Doyle essaya de créer un homme " qui pousse cette méthode aussi loin que possible ${ }^{28}$. » Bell acceptait le compliment, reconnaissant que son élève, Doyle, avait créé : « un homme malin, à l'esprit vif et curieux, mi-médecin, mivirtuose.» Il ajoutait, humblement, que son seul mérite était d'avoir insisté sur la nécessité d'accorder une extrême attention aux moindres

25 Jacques Lacan, « Le Séminaire sur La Lettre volée », Ecrits, Paris, Seuil, 1966, p. 11-61.

${ }^{26}$ Pamela Tytell, "Sherlock Holmes et le cas du Docteur Freud, » Passages du Sujet : Art, Littérature et Psychanalyse: Actes des rencontres de février 1986 à Marseille, Paris/Marseille, Editions du Champ Vallon - Distique et P.U.F., 1987, p. 169-171 (p. 169177). Cet article étudie des relations entre la psychanalyse et le roman policier et les similarités entre les deux médecins-écrivains Arthur Conan Doyle et Sigmund Freud.

${ }^{27}$ Sir Arthur Conan Doyle, Memories and Adventures, Boston, Little Brown \& Co., 1924.

${ }^{28}$ Arthur Conan Doyle (1892), cité par R.L.Green, éd. in The Uncollected Sherlock Holmes, Londres, Harmondsworth, Penguin, 1983, p. 18, cité par Pamela Tytell, « Sherlock Holmes et le cas du Docteur Freud, » op.cit., p. 169-171, 172, 173, 176 
détails. "L’appréciation des différences infimes », écrivait Bell, «se trouve à la base du diagnostic médical. La curiosité insatiable, le sens aigu de l'observation et l'éducation spécialisée du médecin sont également les qualités du détective confirmé29.»

David Shore, producteur exécutif, qui a crée le personnage du Dr. Gregory House, est amateur de l'œuvre d'Arthur Conan Doyle. Le personnage qu'il a crée pour la série médicale télévisée agit exactement comme Sherlock Holmes. Le jeu de mots délibéré autour des noms de famille (house $=$ home) est plus qu'un simple indice. Ainsi, David Shore a ajouté un ingrédient supplémentaire à la recette du succès : de nombreuses références aux œuvres et à la vie d'Arthur Conan Doyle. Ces deux personnages de fiction sont drogués, indifférents envers leurs clients, n'acceptent que les cas les plus intéressants et se servent du même raisonnement et de la même méthode d'enquête liés à la recherche de la vérité. Ce raisonnement dialectique - un rationalisme expérimental ou empirique - est la méthode Socratique du dialogue. House et Holmes cherchent et analysent les indices et le réseau de signifiants, pour construire, expérimenter et puis réfuter ou valider les hypothèses, afin d'aboutir à la vérité, au bon diagnostic et au traitement approprié. House et Holmes excellent dans la construction d'hypothèses, même s'ils ont besoin d'un interlocuteur en face pour en discuter et les réfuter. Sur le site officiel de la série on trouve une citation attribuée à Sherlock Holmes : «When you have eliminated all which is possible, then whatever remains, however improbable, must be the truth ${ }^{30}$." Ainsi, Holmes et House, donnent du sens au morcellement des données en les intégrant à un ensemble fini et solide. Parmi les similarités entre les deux personnages de fiction, on peut remarquer que le docteur Gregory House et Sherlock Holmes sont tous deux musiciens, et ont un seul ami qui s'appelle Dr. James Wilson pour l'un et Dr. John Watson pour l'autre. Le numéro de l'appartement de House, $221 \mathrm{~B}$, est également l'adresse de Holmes à Londres, 221B Baker Street. Et plusieurs épisodes de House, M.D., évoquent Doyle et ses romans. Le patient dans l'épisode pilote s'appelait Rebecca Adler, d'après Irene Adler, un personnage proche de Holmes. A la fin de la deuxième saison, (2.24) l'époux d'une patiente qui s'est suicidée tire sur House. Il s'appelle Moriarity, le nom de l'ennemi de Holmes. Dans la quatrième saison, (4.10) House reçoit une deuxième édition d'Arthur Conan Doyle comme cadeau. Au cours de la cinquième saison, (5.7) House prend ses clés et son Vicodin qui sont posés sur un exemplaire de The Memoirs of Sherlock Holmes d'Arthur Conan Doyle. Et dans un autre épisode, (5.11) House utilise un livre de Joseph Bell - le médecin

29 J.M. Saxby, Joseph Bell : An Appreciation by an Old Friend, Edinburgh, Oliphant, Anderson \& Ferrier, 1913; J. Bell, "The Adventures of Sherlock Holmes: A Review », Bookman, 2, no. 73, 1892, cité par Pamela Tytell, ibid., p. 172-173.

$3^{\circ}$ Voir le site officiel de la série <http://www.housemd-guide.com>, consulté en janvier 2012. 
qui a inspiré le personnage de Sherlock Holmes - pour tromper son équipe. Le livre a été offert par Wilson. Mais ce dernier dit à l'équipe qu'il s'agit d'un cadeau d'une patiente nommée Irene Adler, avant d'avouer être celui qui a offert ce livre de collection à House.

\section{Une nouvelle perception du monde médical}

Il faut enfin évoquer la présence d'un autre ingrédient pour expliquer le succès de cette série : la modification de la perception du monde médical chez certains téléspectateurs. Même s'ils sont mieux informés des symptômes et des traitements de pointe, certaines personnes croient que tout se passe comme dans les séries dont ils sont friands et ont une vision faussée du fonctionnement du monde médical. En effet, House, M.D. et Grey's Anatomy présentent une vision ne correspondant en rien aux standards en vigueur dans la plupart des hôpitaux ; de plus, la valorisation constante du médecin et de sa mission de sauveur de l'humanité souffrante dans ces séries ont amené certains téléspectateurs-patients à croire que ces prouesses imaginaires ou expérimentales - ainsi que ces interventions spectaculaires - existent dans la réalité. Selon Jean-Michel Bader, ces médecins font des miracles à la télévision, mais il est difficile pour les patients d'accepter que leurs vrais praticiens ne puissent pas réaliser les mêmes prouesses ${ }^{31}$. Andrew Holtz pense, lui aussi, que ces fictions incitent certaines personnes à croire qu'ils peuvent demander à leur médecin de bénéficier des thérapies imaginaires vues sur le petit écran ${ }^{32}$. Ceux qui pensent que le monde médical fonctionne de cette façon adaptent la fiction télévisée à leur propre réalité médicale. Tantôt ils exigent un rendez-vous avec un spécialiste dans les vingt-quatre heures, tantôt ils demandent la présence d'un diagnosticien comme Dr. House pendant leur hospitalisation, ou encore ils s'attendent à la même rapidité d'exécution, qu'il s'agisse de l'intervention d'une infirmière ou de la réception immédiate des résultats d'analyses.

Il semblerait que le fait de regarder des séries médicales ait une autre conséquence sur les téléspectateurs. L'étude universitaire de Yinjiao Ye, Professeur de Communication à l'University of Rhode Island, fait sourire quand elle écrit que l'exposition au contenu médical de E.R., Grey's Anatomy ou House, M.D., provoque chez les spectateurs une angoisse excessive concernant leur état de santé et leur vie personnelle. Si Yinjiao Ye veut bien reconnaître que l'on peut apprendre beaucoup sur la médecine en regardant la télévision, elle pense toutefois que les téléspectateurs sont convaincus qu'ils vont tomber malade en attrapant les maladies observées à la télévision. Bref, au lieu de nous rassurer, ces connaissances pointues nous rendraient

${ }^{31}$ Jean-Michel Bader, op.cit.

${ }^{32}$ Andrew Holtz, op.cit. 
plus anxieux et hypocondriaques. Ce phénomène n'est pas nouveau. On a souvent parlé du "syndrome de l'étudiant en médecine », et des " cyberchondriaques", grands consommateurs de sites comme " doctisssimo ", mais je doute fort que les téléspectateurs confondent migraine et cancer du cerveau. Que propose Yinjiao Ye ? Regardez moins la télévision, faites de l'exercice et sortez avec vos amis ${ }^{33}$. Neil Haesler, journaliste au Postmedia News, résumait ces conseils de façon ironique: "The next time you are watching House and he's making medical history reattaching someone's severed foot, put your own shoes on and go for a walk ${ }^{34}$. »
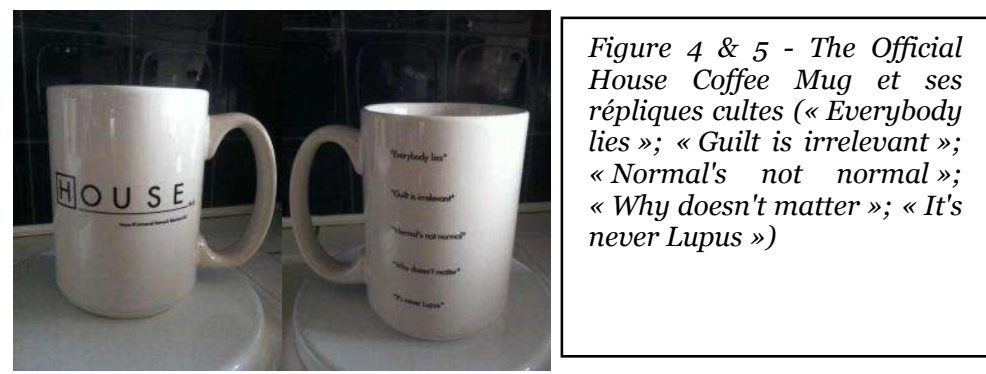

L'amateur des séries médicales - qui lit des journaux américains - peut observer des similarités entre la vie des comédiens, en dehors des plateaux de tournage, et celle des personnages qu'ils incarnent35. A force d’interpréter le médecin boiteux, Hugh Laurie a

33 Yinjiao Ye, «Beyond Materialism: The role of health-related beliefs in the relationship between television viewing and life satisfaction among college students, " Mass Communication and Society, vol. 13, no. 4, septembre 2010, p. 458-478.

34 Neil Haesler, « Medical shows can make you ill: study, " Postmedia News, 1-2 octobre, 2010

$<$ www.canada.com/health/Medical+shows+male+Study/3608468>;

$<$ www.montrealgazette.com/story_print.html?id=3614172\&sponsor=>, consultés en janvier 2012.

$35 \mathrm{Au}$ colloque du Havre, j’ai évoqué d'autres cas où la vie des comédiens et celle des personnages qu'ils incarnent à la télévision se rejoignent. Par exemple, les psychanalystes américains avaient décerné une récompense à la comédienne Lorraine Bracco pour la véracité de son interprétation d'une psychanalyste de fiction, Dr. Melfi, créée par des scénaristes d'Hollywood pour la série The Sopranos. La comédienne avait entrepris une psychanalyse neuf ans avant de jouer dans The Sopranos. Quand Lorraine Bracco faisait la promotion de son livre On the Couch (New York, Putnam, 2006) à la télévision, elle évoquait ses problèmes personnels. Tout comme le personnage qu'elle interprétait dans la série The Sopranos, Lorraine Bracco souffre de dépression. En 2008, la comédienne faisait de la publicité à la télévision pour des médicaments, fabriqués par le laboratoire Pfizer, qui soignent la dépression. Par ailleurs, le personnage du Dr. Melfi souffre d'alcoolisme. Et la comédienne Lorraine Bracco est viticultrice et propriétaire de Bracco Wines, acheté avec l'argent gagné en jouant une alcoolique dans cette série. Pour davantage d'éléments voir l'article publié dans GRAAT (http://www.graat.fr/tv13tytell.pdf) 
des problèmes à la hanche et aux genoux ${ }^{36}$. A terme, ceci pourrait mettre fin à sa présence dans la série. Comme le personnage qu'il interprète à la télévision, Hugh Laurie a la passion des motos, de la littérature et de la musique. Il est même l'auteur d'un roman policier. Pour le lancement de son roman en format poche, les éditions du Seuil ont insisté sur la relation entre le personnage du médecin et le comédien qui écrit. Ainsi, sur les affiches dans le métro parisien, on voyait le visage de Hugh Laurie. A côté de sa photo on pouvait lire : « J'ai une autre maladie, j'écris37. » Côté musique, Hugh Laurie est aux claviers dans le groupe Band from TV Global Charity Trust composé d'acteurs: James Denton (Desperate Housewives), Greg Grunberg (Heroes), Bob Guiney et Bonnie Somerville ${ }^{3}$. Le groupe verse ses cachets aux associations caritatives telles International Myeloma Foundation, Epilepsy Foundation, The National Coalition Against Domestic Violence ou Save The Children. Hugh Laurie joue également du piano et chante dans son propre groupe, qui a sorti un album en avril 201139: une reprise des grands standards du blues des années 1920 et 1930. Le père de Hugh Laurie, ainsi que le père de Lisa Edelstein, qui interprète le Dr. Lisa Cuddy, étaient médecins. Et les comédiens de la série participent aux campagnes pour financer la lutte contre le SIDA et la recherche sur la maladie mentale, notamment auprès de The National Alliance on Mental Illness (NAMI).

En France, un médecin en titre reconnaît faire partie des amateurs de la série House, M.D. Il s'agit de Michel Cymes, qui présente Le Magazine de la Santé (France 5) avec Marina Carrère d'Encausse. A plusieurs reprises il a avoué être un grand admirateur du personnage de Gregory House. En 2009, il a même fait croire qu'il allait jouer comme figurant dans la série. Pour le lancement du site Bonjour-docteur.com en mars 2009, il s'est déguisé en House pour le tournage d'une parodie, allant jusqu'à substituer des comprimés de Viagra à ceux du Vicodin. Sur YouTube, on peut regarder la vidéo réalisée par l'équipe du site Bonjour-docteur.com au moment de son lancement. Je souhaite attirer l'attention sur la phrase qu'on peut lire à la fin de la vidéo : "La santé, c'est pas du cinéma40. " En effet, ceci nous ramène au début de cet article et à la phrase choisie comme titre.

\footnotetext{
${ }^{36}$ «Hugh Laurie: quand la blessure fictive devient la réalité!, » 20 septembre, 2009. $<$ www.people.premiere.fr/News-People/Hugh-Laurie-quand-la-blessure-fictive-devient-laréalité>, consulté en janvier 2012.

37 Hugh Laurie, The Gun Seller, Londres, Random House, 1996 republié par Arrow Books, 2009. Paru en français sous le titre Tout est sous contrôle, trad. de l'anglais par Jean-Luc Piningre, Paris, Sonatine Editions, 2009 ; Paris, Seuil, Points- P 2396, 2010.

38 Voir le site official de «Band From TV Global Charity Trust, » <http://www.bandfromtv.org>, consulté en janvier 2012.

39 Hugh Laurie, Let Them Talk, Warner Music, avril 2011.

${ }_{40}$ "Michel Cymes en docteur House de doctbernardo » <http://www.youtube.com/watch?v=cKs2iFGqHxo\&feature=youtube_gdata_player> consulté en janvier 2012.
} 
Dans les vieux films américains et dans les théâtres, les stades ou les salles de concert aux Etats-Unis, lorsqu'il y a une urgence médicale, quelqu'un s'empare du micro et pose toujours la même question : « Is there a Doctor in the House ${ }^{41}$ ? » Il me semble que le téléspectateur est en droit de se poser cette même question quand il regarde certaines séries médicales à la télévision.

${ }^{41}$ Une partie de cet article a été présentée, sous forme de communications, au cours de trois colloques. «Les Séries Télévisées » au Centre Culturel International de Cerisy-la-Salle (1421 août 2002); Les pièges des nouvelles séries télévisées américaines, mécanismes narratifs et idéologiques à l'Université du Havre (septembre 2009), dont la communication a été publiée sous le titre "Quelques réflexions sur la psychanalyse, l'identification et le réalisme dans les séries télévisées américaines", Les pièges des nouvelles séries américaines :mécanismes narratifs et idéologiques, GRAAT on-line \# 6, décembre 2009 , p. 211-239<http://www.graat.fr/tv13tytell.pdf>; "Les séries télévisées américaines contemporaines : entre la fiction, les faits et le réel, » à l'Université Paris-Diderot (5-7 mai 2011). 